\title{
Neurological manifestations of Ehlers-Danlos syndrome
}

\author{
T. Mathew, S. Sinha, A. B. Taly, G. R. Arunodaya, S. G. Srikanth ${ }^{*}$ \\ Departments of Neurology and *Neuroradiology, National Institute of Mental Health and Neurosciences, Bangalore, India
}

\begin{abstract}
Ehlers-Danlos Syndrome (EDS) is more identified for its cutaneous features but its neurological manifestations have not received the focused attention. Four patients of EhlersDanlos Syndrome (EDS) with neurological manifestations were evaluated for phenotypic data. These four men were from three families and two had consanguineous parentage. The mean age at onset and presentation of neurological symptoms were 10.5 years and 19 years respectively. $\mathrm{Pa}-$ tient 1 presented with bilateral optic atrophy, sensorineural deafness, cerebellar ataxia and neuropathy. Patient 2 had marfanoid habitus, chorea and cerebellar ataxia. Patient 3 had action and percussion myotonia, wasting and weakness of sternocleidomastoid and distal limb muscles. Patient 4 had action myotonia, mirror movements of both hands and neuropathy. MRI of brain showed right parietal polymicrogyria. Neuroaxis involvement at multiple levels in EDS may have prognostic significance.
\end{abstract}

Key words: Ataxia, chorea, Ehlers-Danlos syndrome, mirror movement, myotonia, neurological manifestations, and polymicrogyria.

\section{Introduction}

Ehlers-Danlos syndrome (EDS) is a generalized connective tissue disorder, characterized by skin fragility, skin hyperextensibility and joint hypermobility and may affect any part of the neuroaxis. ${ }^{[1]}$ Cerebrovascular complications are common and other neurological manifestations are rare. ${ }^{[2]}$ We report four patients of EDS with neurological manifestations other than cerebrovascular complications.

\section{Case History}

These four patients clinically diagnosed EDS were evaluated from 1998 to 2002 . Their phenotypic data, family pedigree and imaging features are described.

\section{Patient 1}

A 20-year old man, presented with delayed motor milestones and unsteady gait since early childhood. He was born of a second-degree consanguineous parentage. There was no similar illness in family. He had hyperextensible joints, hyperelastic skin, pes planus, scoliosis, left facial hemiatrophy, primary optic atrophy and sensorineural deafness, distal limb weakness, hyporeflexia, flexor plantar response and impairment of vibration sense in toes and impaired tandem gait. Complete hemogram and routine biochemical tests including serum creatine kinase (CPK) were normal. Nerve conduction (NC) study showed evidence of demyelinating motor-sensory neuropathy. Magnetic resonance imaging (MRI) of brain was normal. Visual evoked responses were not recordable bilaterally. Sural nerve biopsy showed focal loss of large diameter fibres and evidence of demyelination. Diagnosis of EDS with optic atrophy, deafness, demyelinating neuropathy and cerebellar ataxia was considered.

\section{Patient 2}

This 22-year old man manifested with swaying while walking, slurring of speech, involuntary movements and fatigability for 2 years. There was no history of similar illness in the family. He had marfanoid habitus, curly hair, progeria, hyperextensible joints and hyperelastic skin, scanning speech, chorea involving all limbs, in-coordination of extremities and impaired tandem gait. Routine hemogram and biochemical examinations including serum CPK, copper, ceruloplasmin, thyroid function test and CSF analysis were normal. Nerve conduction studies and multimodal evoked responses did not reveal any abnormality. MRI brain showed cerebellar and brainstem atrophy [Figure 1]. Genetic analysis was not performed. A diagnosis of EDS with chorea and cerebellar ataxia was made.

\section{Patient 3}

This 15-year old boy reported with progressive proximal weakness and difficulty in releasing handgrip of 5 years du- 


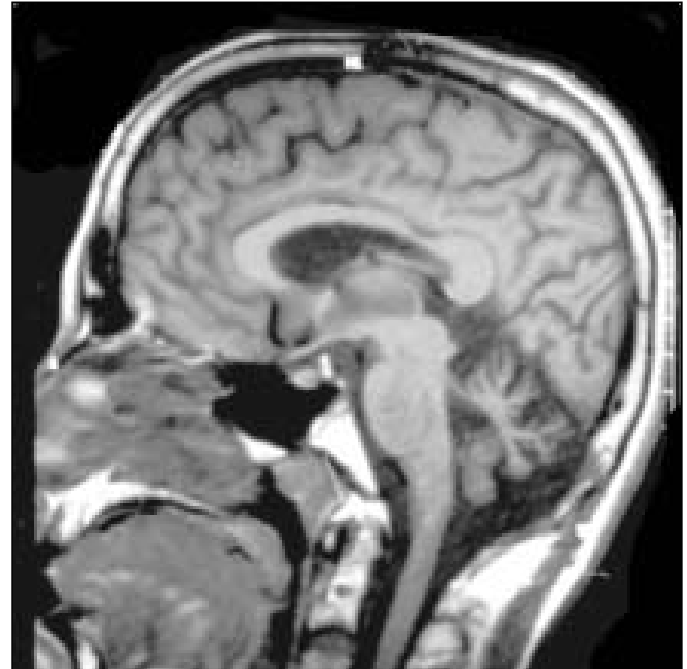

Figure 1: MRI (T1W, sagittal) showing cerebellar and brainstem atrophy (Patient 2)

ration. He was born pre-term (7th month of gestation) to consanguineous parents. His elder brother (Case 4) had similar illness. He had hyperelastic skin [Figure 2A], hyperextensible joints with positive thumb and wrist sign, 'cigarette paper' like scars on the right knee and pes cavus deformity. There was wasting of sternocleidomastoid, forearm and intrinsic muscles of the hand, and hypertrophy of calf and extensor digitorum brevis muscles. Most remarkable was percussion and action myotonia [Figure 2B] in all muscles of the limbs, facial musculature and tongue. Minimal weakness (MRC grade: $4 / 5$ ) was present, but was more in distal muscles. Muscle stretch reflexes were diminished in the upper limbs and normal in the lower limbs. Plantar response was flexor. There was no evidence of frontal baldness, cataract, gynaecomastia or testicular atrophy. Hemogram, routine biochemical tests and serum CPK were normal. NC study was normal. Electromyography revealed a myopathic pattern with typical myotonic discharges. Left biceps muscle biopsy showed features suggestive of mild neurogenic atrophy. A diagnosis of EDS type 1 with an associated myotonic disorder was established.

\section{Patient 4}

A 19 year old boy, elder brother of Patient 3, presented with minimal difficulty in releasing objects from the left hand for 4 years, mild difficulty in standing from sitting position, mirror movements of hands and occasional falls while walking for past 11 years. He had short neck, hypopigmented areas over scalp, keloid scars over shoulder and chest, high arched palate, periodontitis, hyperextensible joints, hyperelastic skin, positive thumb and wrist sign, Cigarette paper scars over both knees, right lower limb wasting and pes planus. He had wasting of bilateral forearm and intrinsic muscles of hand and left hand action myotonia but no percussion hypotonia. Mirror movements in both hands were evident. There were no other deficits. Hemogram and biochemical investigations including

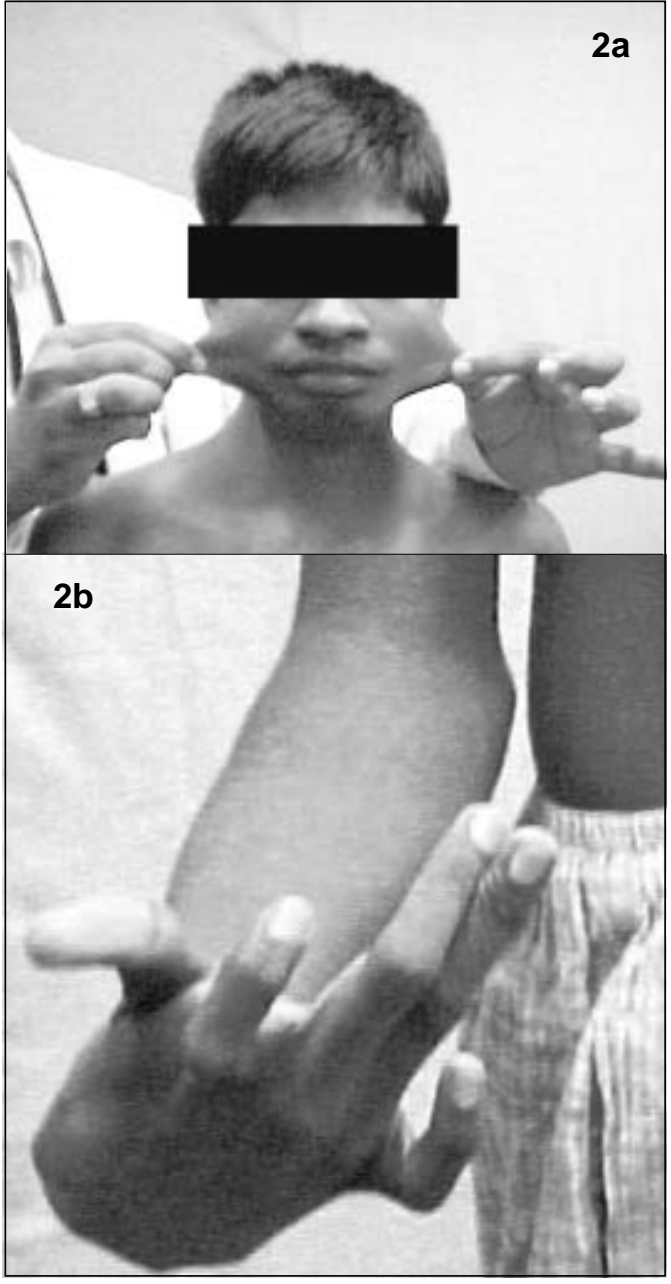

Figure: 2 (A): Hyperelasticity of skin in Ehlers - Danlos syndrome (Patient 3) 1 (B): Action myotonia of hand muscles (Patient 3)

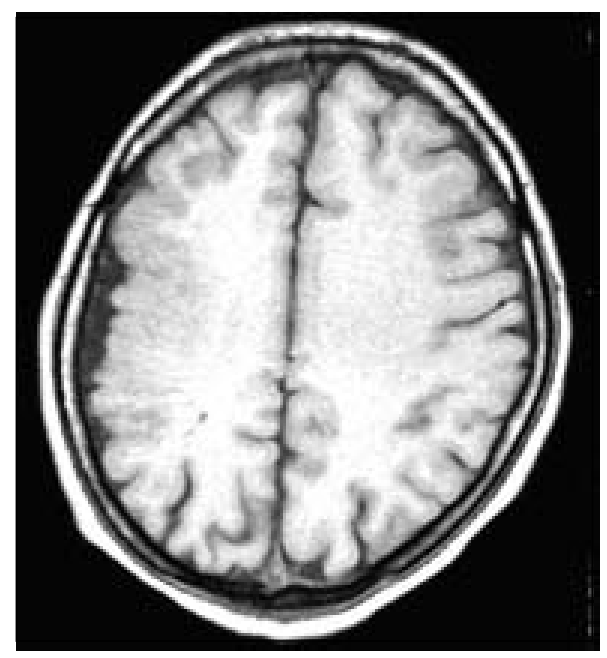

Figure 3: MRI (T1W, axial) showing polymicrogyria of right parietal region (Patient 4 )

Defects in extra cellular matrix proteins have been proposed as a mechanism for arterial aneurysms and EDS IV. ${ }^{[10]}$ Disruption between the extracellular matrix and cytoskeleton 
serum CPK and T3, T4, TSH were normal. NC study showed reduced compound muscle action potential in right median $(5.52 \mathrm{mV})$ and ulnar $(4.94 \mathrm{mV})$ nerves with preserved conduction velocity and normal parameters in the lower limbs. Electromyography revealed myotonic discharges. MRI (brain) revealed right parietal polymicrogyria [Figure 3]. MRI (cervical spine) showed insignificant lateral displacement of odontoid to the left. A diagnosis of EDS with mirror movements, polymicrogyria and axonal neuropathy was considered.

\section{Discussion}

Ehlers-Danlos syndrome constitutes a group of connective tissue disorders characterized by clinical and genetic variability. ${ }^{[3]}$ Mutations in 8 separate genes contribute to these phenotypes. Earlier reports were focused on the skin and ocular abnormalities. The heterogeneity began to be appreciated in the past few decades, from the insights gained from biochemical and molecular genetic studies. ${ }^{[1]}$

Ehlers-Danlos syndrome type 1 has an autosomal dominant inheritance and affected individuals have soft, velvety, hyperextensible skin, joint hypermobility, easy bruisablility and thin, atrophic 'cigarette-paper' scars following trauma. About $50 \%$ of the infants with EDS 1 are born 4 to 8 weeks prematurely because of the fragile fetal membranes as in our Patient 3. ${ }^{[1]}$ Neurological manifestation other than stroke and hypotonia include peripheral neuropathy, ${ }^{[4]}$ epilepsy, ${ }^{[5]}$ neuronal migration disorders, ${ }^{[3,6]}$ Melkerson - Rosenthal syndrome, ${ }^{[7]}$ familial spastic ataxia ${ }^{[8]}$ and dentatopallido luysian atrophy. ${ }^{[9]}$ EDS has a prevalence of 1 in 10,000 persons. It is therefore possible that these neurological illnesses coexist rather than causally linked. ${ }^{[1]}$ In the present series rare manifestations like neuropathy, optic atrophy, deafness, cerebellar ataxia, chorea, myotonia, calf hypertrophy, polymicrogyria and mirror movements were noted. Patient 2 had chorea, ataxia and cerebellar/ brainstem atrophy raising a possibility of cooccurrence of DRPLA. Similarly Patients 3 and 4 had features of myotonia. However genetic analysis could not be carried out in them. causes aberrations in cellular migration. This might result in various congenital malformations of the brain parenchyma like neuronal migration disorders. ${ }^{[5]}$ The extracellular matrix proteins involved may affect collagen or tenascins, a family of glycoproteins expressed in the surface of neurons and glial cells. ${ }^{[6]}$ Mice deficient in small leucine rich proteoglycans develop osteoporosis, EDS, muscular dystrophy and corneal diseases. This may be the link between some cases of muscular dystrophy and EDS. ${ }^{[1]}$

Ehlers-Danlos syndrome may affect all level of neuraxis. A careful search may reveal asymptomatic and unrecognized abnormalities, which offer better explanations for the patient's symptoms and may alter prognosis.

\section{References}

1. Byers PH. Disorders of collagen biosynthesis and structure. In: Scriver CR Beaudet AL, Sly WS, Valle D, editors. The Metabolic and molecular bases of inherited disease. $7^{\text {th }}$ ed. New York: McGraw Hill; 1995;5241-71.

2. Pretorius ME, Butler IJ. Neurologic manifestations of Ehlers-Danlos syndrome. Neurology 1983;33:1087-89.

3. Thomas P, Bossan A, Lacour JP, Chanalet S, Ortonne JP, Chatel M. EhlersDanlos syndrome with subependymal periventricular heterotopias. Neurology 1996;46:1165-67.

4. Muellbacher W, Finisterer J, Mamoli B, Bittner RE, Trautinger F. Axonal neuropathy in Ehlers -Danlos syndrome. Muscle Nerve 1998; 21:972-4.

5. Jacome DE. Epilepsy in Ehlers - Danlos Syndrome. Epilepsia 1999;40:46773.

6. Echaniz-Laguna A, de Saint-Martin A, Lafontaine A L, Tasch E, Thomas P, Hirsh E et al. Bilateral Focal Polymicrogyria in Ehlers - Danlos syndrome. Arch Neurol 2000;57:123-7.

7. Caksen H, Cesur Y, Tombul T, Uner A, Kirimi E, Tuncer O et al. A case of Melkersson-Rosenthal syndrome associated with Ehlers - Danlos syndrome. Genet Couns 2002;13:183-6.

8. Chouza C, Caamano JL, De Medina O, Bogacz J, Oehninger C, Vignale R et al. Familial spastic ataxia associated with Ehlers-Danlos syndrome with platelet dysfunction. Can J Neurol Sci 1984;11:541-549.

9. Sugie K, Nakamuro T, Harada N, Suzumura A, Takayanagi T. A report of two siblings with both maternal dentate-rubro - pallido - luysian atrophy and paternal Ehlers - Danlos syndrome type III. Rinsho Shinkeigaku $1998 ; 38: 233-7$.

10. North KN, Whiteman DAH, Pepin MG, Byers PH. Cerebrovascular complications in Ehlers-Danlos syndrome type IV. Ann Neurol 1995;38:960-4

11. Ameye L, Young MF. Mice deficient in small leucine - rich proteoglycans: novel in vivo model for osteoporosis, Ehlers - Danlos syndrome, muscular dystrophy and corneal disease. Glycobiology 2002;12:107-16

Accepted on 23-05-2005 